${ }^{\circ}$ Entomologica Fennica. 30.IV.1992

\title{
Further information on the oribatid fauna (Acarina, Oribatei) of the northern palearctic region: Ukraina and Czechoslovakia
}

\author{
Eero Karppinen, V. V. Melamud, Ladislav Miko \& D. A. Krivolutsky
}

Karppinen, E., Melamud, V. V., Miko, L. \& Krivolutsky, D. A. 1992: Further information on the oribatid fauna (Acarina, Oribatei) of the northern palearctic region: Ukraina and Czechoslovakia. — Entomol. Fennica 3:41-56.

A list of the oribatids of Ukraina and Czechoslovakia, with find localities, has been compiled from published reports and new collecting data. Altogether 453 species or subspecies are now known from Ukraina and 186 from Czechoslovakia.

Eero Karppinen, Zoological Museum, University of Helsinki, SF-00100 Helsinki, Finland

V. V. Melamud, Museum of Natural History, Ukrainian Academy of Sciences, Lvov, Ukraina

Ladislav Miko, Centrum Biologicko-Ekologickych Vied SAV, Pobočka pre ekologiu palnohospodarskej krajiny. 04000 Ko šice, Kukorelliko 10, Československo

D. A. Krivolutsky, Institute of Evolutionary Morphology and Ecology of Animals, Russian Academy of Sciences, Moscow, Russia

\section{Introduction}

The abundance of oribatid mites in the soil, together with the good preservation properties and frequent species-specific characters of their armours, makes oribatids an important tool for biostratigraphical research.

To use oribatid data effectively in paleoecology, paleogeography and biostratigraphy, it is necessary to know the ecology and present distribution of the species. Previous to our joint investigations, no such data had been compiled, which limited the use of oribatids in paleogeography and in the interpretation of biostratigraphical data.

In 1973 E. Karppinen and D. A. Krivolutsky initiated faunistical investigations in the eastern part of the northern palearctic and published faunistic-ecological lists (see below) as well as a review of the published data concerning western Europe (Karppinen et al. 1979). The species list for eastern and northern Europe (Karppinen \& Krivolutsky 1982) comprises 526 species, of which about 100 have not been reported earlier from the region. In Siberia, 555 species have been found (Golosova et al. 1983). In addition, we have found an earlier, unknown pre-glacial fauna of oribatid mites (Krivolutsky et al. 1984), but a large part of this material is still unpublished.

The arid zones of the palearctic (Karppinen et al. 1986), and of the Crimea and the Caucasus have also been studied (Karppinen et al. 1987). It was necessary to include the last-mentioned regions (which are situated outside the borders of the northern palearctic, the southern border of which we define as the southern border of ever- 


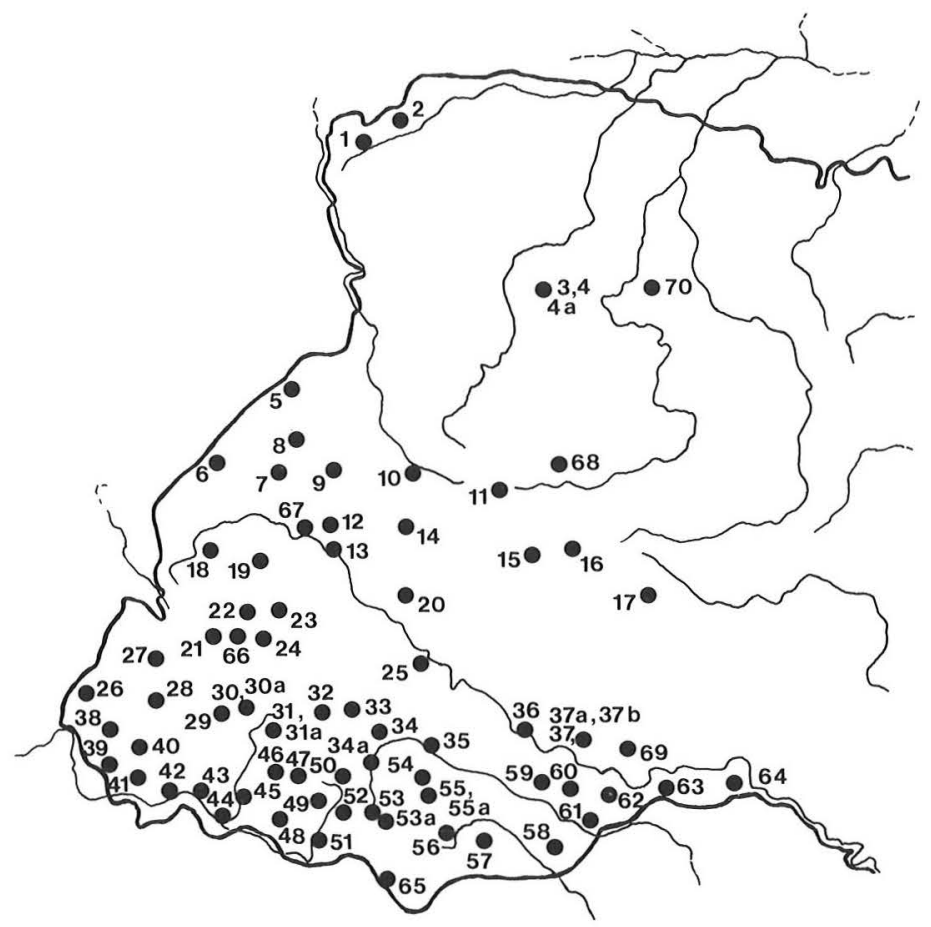

Fig. 1. Collection localities in Ukraina (for key to numbers, see list of localities). green forests in Europe and Siberia), because no earlier data were available for this area, and many oribatid species abundant in the Crimea and the Caucasus are distributed in fields, sandy soils and meadows on south-facing valley slopes as far north as Moscow and southern Sweden. Without knowledge of the fauna of the Crimea and the Caucasus it is impossible to understand the patterns of geographical distribution in the other areas.

This last list, comprising 496 species or subspecies, 453 from Ukraina and 186 from Czechoslovakia, completes the previously published lists of oribatid mites of the northern palearctic region (Karppinen \& Krivolutsky 1982, Golosova et al. 1983, Karppinen et al. 1986 and Karppinen et al. 1987).

In addition, while compiling the faunistical lists, the authors (E. Karppinen, D. A. Krivolutsky, L. D. Golosova, E. V. Gordeeva, Z. Tarba, M. P. Poltavskaja, U. Schtanchaeva) found and described more than 60 new species of oribatid mites.

The systematic arrangement follows Balogh (1972).

\section{List of localities}

In the list of species, and in the maps (Figs. 1 and 2 ), the localities are indicated with their numbers.

\section{Ukraina}

1 Shatsk: Kazakov 1981, Melamud (unpubl.).

2 Tur: Kazakov 1981, Melamud (unpubl.).

3 Volynskaya obl.: Grishina \& Sergienko 1978, Sitnikova 1975, Yaroshenko 1978, Shaldybina 1981, Sergienko 1984.

4 Volynskaya obl.: Yaroshenko (unpubl.).

4a Kivertskii r-n: Yaroshenko (unpubl.).

5 Rava-Russkaya: Dyrdowska 1931.

6 Mostiskii r-n: Kulczynski 1902.

7 Gorodotskii r-n: Zgerskaya 1980, Zgerskaya \& Senyk 1975.

8 Yavorovskii r-n: Zgerskaya \& Senyk 1975, Melamud (unpubl.).

9 Lvov (+ Nesterov): Zgerskaya \& Senyk 1975, Dyrdowska 1931, Melamud (unpubl.), Sergienko 1987. 
Fig. 2. Collection localities in Czechoslovakia (for key to numbers, see list of localities).

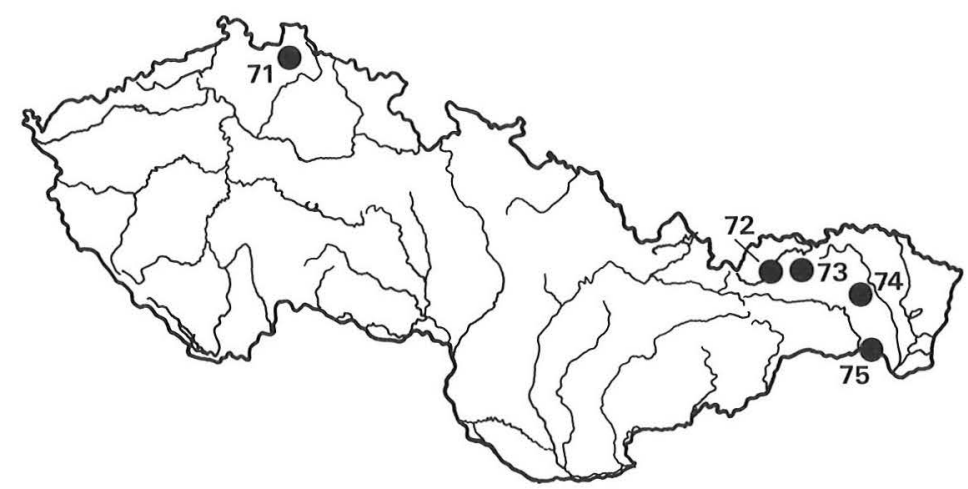

10 Lopatin (+ Chevone): Melamud (unpubl.).

11 Pochaev: Melamud (unpubl.).

12 Kologory: Melamud (unpubl.).

13 Hodorov: Melamud (unpubl.), Yavornitskii et al. 1984.

14 Chemerintsy: Melamud (unpubl.).

15 r. Seret: Dyrdowska 1931.

16 Gribov: Dyrdowska 1931.

17 Khmelnitskaya obl.: Sevastanov 1970.

18 Malinov: Melamud (unpubl.).

19 Truskavets: Zgerskaya \& Senyk 1975, Melamud (unpubl.)

20 Burshtyn: Melamud (unpubl.).

21 Klimets: Melamud (unpubl.).

22 Dubina: Melamud (unpubl.).

23 Rozgirche: Melamud (unpubl.).

24 Morshin: Melamud (unpubl.), Sergienko 1985, 1987a, 1987b.

25 Ivano-Frankovsk: Melamud (unpubl.), Yaroshenko (unpubl.).

26 Uzhgorod: Kazakov 1986, Melamud (unpubl.).

27 Lumshory: Kazakov (unpubl.), Melamud (unpubl.).

28 Chinadievo: Kurcheva 1970, (unpubl.).

29 Soimy (g. Kuk): Kazakov (unpubl.), Melamud (unpubl.).

30 Toryn: Melamud (unpubl.), Sergienko 1987.

30a Sinivir: Kazakov (unpubl.), Melamud (unpubl.).

31 pol. Dervaika: Kazakov (unpubl.), Melamud (unpubl.)

31a Kolocheva: Kazakov (unpubl.), Melamud 1986, (unpubl.)

32 Osmoloda: Kazakov (unpubl.), Melamud (unpubl.).
33 Bogorodchanskii r-n: Melamud (unpubl.).

34 Hadvornyanskii r-n: Melamud (unpubl.), Yaroshenko (unpubl.).

34a Hadvornyanskii r-n: Melamud (unpubl.), Yaroshenko (unpubl.).

35 Kolomyya: Yaroshenko (unpubl.).

36 Zalishchiki: Sergienko 1985, 1987a

37 Komendyany: Melamud (unpubl.).

37a Germakovka: Melamud (unpubl.).

37b Chemerovtsy: Grishina \& Sergienko 1978

38 Mukachevo: Bulanova-Zakhvatkina 1967, Kurcheva 1973, Melamud (unpubl.), Sergienko 1987.

39 Beregovo: Melamud (unpubl.).

40 Zakarpatskaya obl : Bulanova-Zakhvatkina 1965, 1967, 1975, Krivolutsky 1975, Polonchik \& Fasulati 1964, Sergienko 1984, 1987a, Sitnikova 1975, Shaldybina 1981.

41 Shalanki: Melamud (unpubl.).

42 Vinogradovo: Melamud (unpubl.).

43 Hust: Melamud (unpubl.).

44 Novoborovo: Melamud (unpubl.).

45 Uglya: Melamud 1986 (unpubl.).

46 Sirokii lug: Melamud (unpubl.).

47 Ust-Chernaya: Kurcheva 1970 (unpubl.), Melamud (unpubl.).

48 Neresnitsa: Melamud (unpubl.).

49 Chernaya Tisa (g. Stig): Kazakov 1985, Melamud (unpubl.).

50 Bystritsa: Melamud (unpubl.).

51 Kostilovka: Melamud (unpubl.).

52 Rahovskii r-n: Yaroshenko (unpubl.).

53 Vorohta: Kazakov 1981, 1985, (unpubl.), Melamud (unpubl.), Rudishin \& Sergienko (unpubl.), Sergienko (unpubl.), Yaroshenko (unpubl.). 
53a g. Pop-Ivan: Melamud (unpubl.).

54 Kosov: Kazakov (unpubl.), Melamud (unpubl.).

55 Yavorov: Yaroshenko (unpubl.).

55a Kuty: Yaroshenko (unpubl.).

56 Putilskii r-n: Yaroshenko (unpubl.).

57 Starozhenetskii r-n: Yaroshenko (unpubl.).

58 Glybokskii r-n: Yaroshenko (unpubl.).

59 Kitsmanskii r-n: Yaroshenko (unpubl.).

60 Zastavnetskii r-n: Yaroshenko (unpubl.).

61 Novoselitskii r-n: Yaroshenko (unpubl.).

62 Hotinskii r-n: Yaroshenko (unpubl.).

63 Kelmenetskii r-n: Yaroshenko (unpubl.).

64 Sokiryanskii r-n: Yaroshenko (unpubl.).

65 Burkut (g. Chivchin): Kazakov 1985, (unpubl.), Melamud (unpubl.).

66 Grebenov: Kazakov (unpubl.), Melamud (unpubl.), Sergienko 1987.

67 Nikolaev: Melamud (unpubl.).

68 Zalestsy: Melamud (unpubl.).

69 Kamenets-Podolsk: Melamud (unpubl.).

70 Rovanskaya obl.: Sergienko 1984.

\section{Czechoslovakia}

71 Hamr na Jezere (N. Bohemia): Miko (unpubl.).

72 Levoceké vrchy, Tichy Potok: Miko (unpubl.).

73 Čergovské pohoris - Majdan: Miko (unpubl.).

74 Slanskě vrchy, Kokošovce-Sigord: Miko (unpubl.).

75 Mala Izra: Miko (unpubl.).

\section{List of species}

PALAEACARIDAE Grandjean, 1932

Palaeacarus Trägårdh, 1932

- P. hystricinus Trägårdh, 1932: 47

APHELACARIDAE Grandjean, 1954

Aphelacarus Grandjean, 1932

- A. acarinus (Berlese, 1910): 74

MESOPLOPHORIDAE Ewing, 1917

Mesoplophorus Berlese, 1904

- M. pulchra Sellnick, 1928: 4

\section{PHTHIRACARIDAE Perty, 1841}

Archiphthiracarus Balogh \& Mahunka, 1979

- A. anonymus (Grandjean, 1933): 4, 23, 40, 43

- A. lanatus (Feider \& Suciu, 1957): 4, 53

- A. ligneus (Willmann, 1931): 22, 24, 34, 34a, 38,43

- A. pilosus Sergienko, 1987: 24, 30, 38, 66

- A. variabilis Balogh \& Mahunka, 1979: 71

Hoplophthiracarus Jacot, 1933

- H. minus (Krivolutsky, 1965): 4

- H. pavidus (Berlese, 1913): 2, 10, 21, 28, 47, 53, 57

Phthiracarus Perty, 1841

- P. borealis (Trägårdh, 1910): 5, 9, 11, 15, 16, 68

- P. conformis Sergienko, 1987: 9, 24

- P. crenophilus Willmann, 1951:74

- P. elemans Aoki, 1982: 4, 58

- P. ferrugineus (C.L.Koch, 1841): 9, 19, 21, 23, 24, 27, 29, 31a, 39, 41, 45-48, 71

- P. flexisetosus Parry, 1979: 74

- P.globosus (C.L.Koch, 1841): 8, 34, 34a, 40, 56, 61, 67

- P. italicus (Oudemans, 1906): 5, 9, 11, 15, $16,24,27,58,66,68$

- P. laevigatus (C.L.Koch, 1841): 22-24, 27, 30, 40, 41, 46, 48, 64

- P. lentulus (C.L.Koch, 1841): 7, 8, 12, 14, 23-25, 39, 40, 47, 56, 58, 68, 74

- P. longulus (C.L.Koch, 1841): 1, 2, 8-10, 1214, 18-24, 26, 27, 29, 31a, 37, 37a, 38, 39, $41,43-47,54,66,67$

- P. nitens (Nicolet, 1855): 2, 8-14, 18, 21-24, 27, 30a, 31, 31a, 32, 33, 37, 37a, 42, 43, 4750, 53, 53a, 54, 65, 66

- P.piger (Scopoli, 1763): 3, 4, 53

- P. spadix Niedbala, 1983: 1, 2, 8, 9, 12, 13, 19, 21, 23-27, 29, 30a 31, 31a, 32, 34a, 39, $41,43,45-51,53,53 a, 54,65,66$

Steganacarus Ewing, 1917

- S. applicatus (Sellnick, 1920): 21, 23, 66, 71

- S. carinatus (C.L.Koch, 1841): 1-5, 7-13, 15, 18-24, 26-29, 30a, 33, 34, 34a, 38, 39, $41-45,47,48,53,56-58,66,67,74,75$

- S. magnus (Nicolet, 1855): 3, 4, 34, 34a, 73

- S. ochraecus Niedbala, 1893: 36

- S. pulcherrimus (Berlese, 1887): 4, 8, 15, 16, $19,24,28,39,42,53,56-64$

- S. punctulatus Sergienko, 1985: 8, 9, 13, 19, 
$21,23,24,26,27,30 \mathrm{a}, 32,36,37 \mathrm{a}, 41,46$, 49, 50, 53, 53a, 54, 66

- S. serratus Feider \& Suciu, 1957: 24, 36

- S. spinosus Sellnick, 1920: 71

- S. striculus (C. L. Koch, 1836): 1, 2, 4, 8, 9, 12-14, 18, 21-27, 29, 30, 30a, 31, 31a, 32, $34,34 \mathrm{a}, 37,37 \mathrm{a}, 38,39,41,43,44,46-49$, 53, 53a, 54, 56-58, 60-66, 68, 71, 73, 75

ORIBOTRITIDAE Grandjean, 1954

Mesotritia Forsslund, 1963

- M. grandjeani Feider \& Suciu, 1957: 30a, 40, $47,50,53$

Oribotritia Jacot, 1924

- O. decumana (C. L. Koch, 1836):28, 47

EUPHTHIRACARIDAE Jacot, 1930

Euphthiracarus Ewing, 1917

- E. cribarius (Berlese, 1904): 3, 4, 6, 34, 36, $38,47,64,71$

- E. monodactylus (Willmann, 1919): 8, 9, 13, $18,21,23-25,27,29,30,30 \mathrm{a}, 31-33,36$, $43-51,53,54,62,63,66$

- E. reticulatus (Berlese, 1913): 21, 23, 27, 44, 46, 47, 53, 66

Microtritia Märkel, 1964

- M. minima (Berlese, 1904): 8, 23, 33, 47, 71

Rhysotritia Märkel \& Meyer, 1959

- R. ardua (C. L. Koch, 1841): 4, 5, 9, 12, 15, 23-25, 34, 34a, 35, 39, 45, 48, 52, 53, 55, 55a, 56, 57, 67, 71

- $\quad$ R. duplicata (Grandjean, 1953): 3, 4, 8, 34, 34a, 35, 52, 55, 55a, 71, 75

- R. loricata (Rathke, 1799): 38

HyPOCHTHONIIDAE Berlese, 1910

Hypochthonius C. L. Koch, 1836

- H. luteus Oudemans, 1917: 7-9, 18, 19, 23, 26, 31a, 38, 43-45, 47, 48, 63, 64, 66, 70, 75

- H. rufulus C. L. Koch, 1836: 1, 3, 8, 9, 13, 14, 18, 22, 24, 26, 31a, 32, 34a, 38, 39, 42, 44, 46, 60, 63, 68, 69, 71, 75

- H. rufulus ssp. europaeus Krivolutsky, 1965: 4, 8,63

ENIOCHTHONIIDAE Grandjean, 1947

Hypochthoniella Berlese, 1910

- H. minutissima (Berlese, 1904): 4, 8, 13, 23, $26,27,29,43,44,48,51,64,71$
COSMOCHTHONIIDAE Grandjean, 1947

Cosmochthonius Berlese, 1910

- C. lanatus (Michael, 1885): 74

- C. ponticus Gordeeva, 1980: 24

BRACHYCHTHONIIDA Balogh, 1943

Brachychochthonius Jacot, 1938

- B. cricoides Weis-Fogh, 1948: 8, 70

- B. jugatus Jacot, 1938: 38

- B. rostratus (Jacot, 1936): 47,70

- B. suecicus Forsslund, 1942: 8, 70, 71

Brachychthonius Berlese 1910

- B. berlesei Willman, 1928: 3, 40, 70, 71

- B. immaculatus Forsslund, 1942: 8, 70, 71

- B. impressus Morizt, 1976: 71

- B. jacoti Evans, 1952:28

Eobrachychthonius Jacot, 1936

- E. latior (Berlese, 1910): 47

- E. mooseri (van der Hammen, 1950): 40

Liochthonius van der Hammen, 1959

- L. alpestris (Forsslund, 1958): 23, 32, 70

- L. evansi (Forsslund, 1958): 8

- L. furcillatus (Willman, 1942): 75

- L. horridus (Sellnick, 1928): 8, 47, 53, 70

- L. hystricinus (Forsslund, 1942): 71

- L. lapponicus (Trägårdh, 1910): 3, 40, 70

- L. muscorum Forsslund, 1964: 13, 23, 34a

- L. perpusillus (Berlese, 1910): 3, 4, 8, 13, 17 , 18, 23, 28, 32, 47, 70

- L. propinquus Niedbala, 1972: 8, 70

- L. sellnicki (Thor, 1930): 3, 47, 70

Neobrachychthonius Moritz, 1976

- N. magnus Moritz, 1976: 71

Poecilochthonius Balogh, 1943

- P. italicus (Berlese, 1910): 13

- P. spiciger (Berlese, 1910): 74

Sellnickochthonius Krivolutsky, 1964

- S. zelawaiensis (Sellnick, 1928): 3, 8, 28, 30a, 40,70

Synchthonius van der Hammen, 1952

- S. crenulatus (Jacot, 1938): 32

- S. elegans Forsslund, 1957: 70

LOHMANNIIDAE Berlese, 1916

Tamnacarus Grandjean, 1950

- T. deserticola (Grandjean, 1934): 34 
EULOHMANNIIDAE Grandjean, 1931

Eulohmannia Berlese, 1910

- E. rigabai Berlese, 1910: 13, 21, 27, 29, 31a, $34 \mathrm{a}, 47,66,74$

PERLOHMANNIDAE Grandjean, 1954

Perlohmannia Berlese, 1916

- P. dissimilis (Hewitt, 1908): 24, 29, 31a, 34, 47,48

EPILOHMANNIDAE Oudemans, 1923

Epilohmannia Berlese, 1910

- E. cylindrica (Berlese, 1904): 24

Collohmannidae Grandjean, 1958

Collohmannia Sellnick, 1922

- C. gigantea Sellnick, 1922: 21, 23, 41, 44, 48

NOTHRIDAE Berlese, 1896

Nothrus C.L. Koch, 1836

- N. anauniensis Canestrini \& Fanzago, 1877: $1,3,8,9,13,18,23,24,26,27,31$ a, 33, 39, 42-46, 48, 54, 66, 70, 74

- N. biciliatus C.L. Koch, 1841: 3-5, 8, 9, 16, 24, 34a, 47, 53, 60-62, 67, 69

- N. borussicus Sellnick, 1929: 8, 27, 29, 31a, 34a, 47, 49, 51, 53, 53a, 56, 65, 70

- N. palustris C.L. Koch, 1839: 3, 8, 9, 13, 15, $16,18,21,23-25,29,34 a, 38,40,46,47,56$, $66,70,71,74$

- N. parvus Sitnikova, 1975: 21, 31, 50

- N. pratensis Sellnick, 1929: 5, 9, 15, 16, 53

- N. silvestris Nicolet, 1855: 1-4, 8, 9, 18, 28, 38-40, 47, 53, 56-64, 70, 71

CAmisiIDAE Oudemans, 1900

Camisia van Heyden, 1826

- C. biurus (C.L. Koch, 1839): 3, 4, 65, 71

- C. biverrucata (C.L. Koch, 1839): 3, 23, 27, 31, 31a, 32, 53, 65

- C. horrida (Hermann, 1804): 4, 15, 16, 34, 71

- C. lapponica Trägårdh, 1910: 31, 32, 53, 65

- C. segnis (Hermann, 1804): 3, 5, 9, 15, 16, 38,70
- C. spinifer (C.L. Koch, 1836): 3-5, 9, 15-17, 26, 31, 31a, 42, 43, 46, 53, 64, 65, 70, 71

Heminothrus Berlese, 1913

- H. longisetosus Willmann, 1925: 71

- H. paolianus Berlese, 1913: 17

- H. targionii (Berlese, 1885): 8, 61, 67, 70

- H. thori (Berlese, 1904): 8, 22, 53

Neonothrus Forsslund, 1955

- N. humicola Forsslund, 1955: 31, 38, 47, 53

Platynothrus Berlese, 1913

- P. grandjeani Sitnikova, 1975: 8, 9, 13, 21, 22, 26, 29 30a, 31a, 33, 39, 42-44, 48, 53, 67

- P. peltifer (C.L. Koch, 1839): 2-4, 7-9, 13, 17-19, 21-24, 28, 29, 30a, 31a, 32, 34a, 38$40,46,47,49,51,53,55,59-62,65-67,70$, 71,74

TRHYPOCHTHONIIDAE Willmann, 1931

Trhypochthonius Berlese, 1904

- T. cladonicola (Willmann, 1919): 3

- T. tectorum (Berlese, 1896): 1-4, 9, 25, 35, $43,51,72$

Trhypochthoniellus Willmann, 1928

- T. setosus Willmann, 1928: 34

MALACONOTHRIDAE Berlese, 1916

Malaconothrus Berlese, 1904

- M. gracilis van der Hammen, 1952: 3, 7, 8, $13,18,22,24,27,31,32,46,48,50,53,71$, 75

- M. punctulatus van der Hammen, 1952: 3

- M. pygmaeus Aoki, 1969: 13, 59

Trimalaconothrus Berlese, 1916

- T. glaber (Michael, 1888): 8,13, 53, 71, 75

- T. novus (Sellnick, 1921): 3, 8

- T. tardus (Michael, 1888): 5, 9, 13

NANHERMANNIIDAE Sellnick, 1928

Nanhermannia Berlese, 1913

- N. comitalis Berlese, 1916: 7,8

- N. coronata Berlese, 1913: 2, 8, 13, 18, 29, 31, 31a, 32, 49, 53, 71, 75

- N. elegantula Berlese, 1913: 7, 8, 19, 28, 31, 38

- N. komareki Kunst, 1956: 40, 47

- N. nana (Nicolet, 1855): 3, 4, 7-9, 13, 15, 16, 19, 21-27, 29, 34, 34a, 35, 38, 39, 43-45, 47, $48,53,55,55 \mathrm{a}, 56-58,66,67,71,74,75$ 
HERMANNIIDAE Sellnick, 1928

Hermannia Nicolet, 1855

- H. convexa (C.L. Koch, 1840): 5, 9, 15, 16, 40,71

- H. gibba (C.L. Koch, 1840): 2, 13, 21, 23, 24, 27, 29, 30a, 31, 31a, 32-34, 34a, 40, 45-47, $49,50,53,65,66,71,74$

- H. scabra C.L. Koch, 1879: 71

HERMANNIELLIDAE Grandjean, 1934

Hermanniella Berlese, 1908

- H. dolosa Grandjean, 1931: 4, 8, 9, 13, 28, $29,38,39,41-43,47,48,59-62,74$

- H. granulata (Nicolet, 1855): 3, 7-9, 28, 38

\section{LIODIDAE Grandjean, 1954}

Liodes von Heyden, 1826

- L. theleproctus (Hermann, 1804): 1, 3, 4, 15$17,60,62$

Platyliodes Berlese, 1917

- P. longisetosus Sitnikova, 1975: 57

- P. scaliger (C.L. Koch, 1840): 3, 4, 8, 13, 15-17, 55, 55a, 74

Poroliodes Grandjean, 1934

- P. farinosus (C.L. Koch, 1840): 14, 38, 46, $47,49,53$

GYMNODAMAEIDAE Grandjean, 1954

Allodamaeus Banks, 1947

- A. femoratus (C.L. Koch, 1840): 3, 4

- A. starki Bulanova-Zakhvatkina, 1967: 4, 15

Gymnodamaeus Kulczynski, 1902

- G. bicostatus (C.L. Koch, 1836): 3, 4, 6, 13, $45,46,56,74$

Plateremaeidae Trägårdh, 1931

Pedrocortesia Hammer, 1958

- P. inaequalis Balogh \& Mahunka, 1965: 8

LICNODAMAEIDAE Grandjean, 1954

Licnodamaeus Grandjean, 1931

- L. undulatus (Paoli, 1908): 34a

DAMAEIDAE Berlese, 1896

Damaeus C.L. Koch, 1836

- D. gracilipes (Kulczynski, 1902): 6, 74

- D. onustus C.L. Koch, 1841: 41, 66, 67
Hypodamaeus Bulanova-Zakhvatkina, 1957

- H. auritus (C.L. Koch, 1840): 8, 9, 21, 23, 26, 31a, 44-47, 66, 74

- H. crispatus (Kulczynski, 1902): 6, 74

- H. interlamellaris Willmann, 1931: 17

- $H$. riparius (Nicolet, 1855): 3, 4, 8, 9, 13, 18, $19,23,25,34,34 \mathrm{a}, 35,38,52,55,55 \mathrm{a}, 56-59$

- H. smirnovi Bulanova-Zakhvatkina, 1957: 8, 23, 29, 31, 31a, 32, 34a, 49, 51, 53, 65

- H. tauricus Bulanova-Zakhvatkina, 1957: 51

Epidamaeus Bulanova-Zakhvatkina, 1957

- E. pseudotatricus Bulanova-Zakhvatkina, 1957: 13

- E. setiger (Kulczynski, 1902): 29, 31a, 43, $49,53,65$

- E. tatricus (Kulczynski, 1902): 40

Paradamaeus Bulanova-Zakhvatkina, 1957

- P. clavipes (Hermann, 1804): 15, 16, 71, 74

Spatiodamaeus Bulanova-Zakhvatkina, 1957

- S. boreus Bulanova-Zakhvatkina, 1957: 26

- S. fageti Bulanova-Zakhvatkina, 1957: 8, 13, 26, 33, 42, 44

- S. subverticillipes Bulanova-Zakhvatkina, 1957: 4, 34

- S. verticillipes (Nicolet, 1855): 8, 9, 71

BELBIDAE Willmann, 1931

Belba von Heyden, 1826

- B. corynopus (Hermann, 1804): 13, 19, 23, 24, 26, 27, 29, 31a, 34a, 38, 41-43, 45-49, $53,59,61-63,66,69,72$

- B. dubinini Bulanova-Zakhvatkina, 1962: 55

- B. montana (Kulczynski, 1902): 47

- B. pseudocorynopus Märkel \& Mayer, 1960: 71

- B. sellnicki Bulanova-Zakhvatkina, 1962: 55, $55 \mathrm{a}$

Metabelba Grandjean, 1836

- M. papillipes (Nicolet, 1855): 2-4, 8, 13, 18, 21, 23, 24, 26, 27, 29, 30, 30a, 31, 31a, 34, 34a, 36, 37a, 39-45, 47, 48, 55-58, 66, 69, 72

- M. propexa (Kulczynski, 1902): 2, 9, 23, 24, 28, 31, 31a, 32, 33, 34a, 38, 46, 47, 49, 50, $53,53 \mathrm{a}, 65$

- M. pulverulenta (C.L. Koch, 1840): 4, 5, 7-9, 13, 15-17, 19, 21, 23, 26-29, 31, 31a, 32-34, $34 a, 38,41,43,45-47,53,53 a, 62-64,66$, 67,74

- M. rohdendorfi Bulanova-Zakhvatkina, 1965: $9,14,21,23,29,33,34 a, 43-46,66$ 
Metabelbella Bulanova-Zakhvatkina, 1957

- M. macrochaeta Bulanova-Zakhvatkina, 1967: 21, 29, 31, 31a, 32, 43, 49, 53, 65

- M. soror Bulanova-Zakhvatkina, 1965: 26

Subbelba Bulanova-Zakhvatkina, 1967

- S. partiocrispa (Bulanova-Zakhvatkina, 1957): 13, 21, 22, 24, 26, 27, 29, 31, 31a, 32, $33,39,42,46,49,50,53,66,68$

BELbodAmAeIDAE Bulanova-Zakhvatkina, 1967

Belbodamaeus Bulanova-Zakhvatkina

- B. tuberculatus Bulanova-Zakhvatkina, 1960: 38

Damaeobelba Sellnick, 1928

- D. minutissima (Sellnick, 1920): 33, 34a, 46, 50

Hungarobelba Balogh, 1943

- H. visnyai (Balogh, 1938): 72

Porobelba Grandjean, 1936

- P. spinosa (Sellnick, 1920): 71, 74, 75

Cepheidae Berlese, 1896

Cepheus C.L. Koch, 1836

- C. brachiatus Sitnikova, 1975: 56

- C. cepheiformis (Nicolet, 1855): 3, 4, 8, 13, 31a, 38, 47, 56, 71

- C. dentatus (Michael, 1888): 40, 71, 74, 75

- C. grandis Sitnikova, 1975: 21, 31, 32, 34, $40,45,50,53,57,67$

- C. latus C.L. Koch, 1836: 3, 21, 31a, 32, 33, $34 \mathrm{a}, 40,53$

Conoppia Berlese, 1908

- C. microptera (Berlese, 1885): 2, 9, 23, 27 , 30, 30a, 31a, 32, 33, 34a, 39, 40, 47, 49-51, $53,53 \mathrm{a}, 65$

Tritegeus Berlese, 1913

- T. bisulcatus Grandjean, 1953: 8, 21, 23, 45, 46, 48, 51, 55, 57, 66

- T. brevisetosus Sitnikova, 1975: 4

MicrozetidAe Grandjean, 1936

Nellacarus Grandjean, 1936

- N. asiaticus Krivolutsky, 1967: 57

\section{CTENOBElbidAe Grandjean, 1965}

Ctenobelba Balogh, 1940

- C. pectinigera (Berlese, 1910): 7, 8, 24, 38

- C. pilosella Jeleva, 1962: 4, 13, 26, 41-45, 48,61
EREMULIDAE Grandjean, 1965

Eremulus Berlese, 1908

- E. flagellifer Berlese, 1908: 57

DAMAEOLIDAE Grandjean, 1965

Damaeolus Paoli, 1908

- D. asperatus (Berlese, 1904): 21, 31a, 39, 45, 62,69

Fosseremus Grandjean, 1954

- F. laciniatus (Berlese, 1905): 9, 13, 47, 62

EREMOBELBIDAE Balogh, 1961

Eremobelba Berlese, 1908

- E. geographica, 1908: 26

EREMAEIDAE Sellnick, 1928

Eremaeus C.L. Koch, 1836

- E. fossulatus Kunst, 1959: 40, 55

- E. hepaticus C.L. Koch, 1836: 2, 8, 13, 15, 18, 21, 23, 24, 26-29, 31a, 33, 34a, 37a, 38, $39,41-48,50,53,66,72-75$

- E. oblongus C.L. Koch, 1936: 3-5, 8, 9, 15, $16,47,55 \mathrm{a}$

- E. silvestris Forsslund, 1956: 1, 2, 6, 38, 53, 65,72

- E. triglavensis Tarman, 1958: 4

Tricheremaeus Berlese, 1908

- T. nemossensis Grandjean, 1954: 34

ZetorChESTIDAE Michael, 1898

Zetorchestes Berlese, 1888

- Z. falzonii Coggi, 1898: 74

- Z. micronychus (Berlese, 1883): 3-5, 9, 13, $15,16,26,28,38,42,58$

TENUIALIDAE Jacot, 1929

Hafenrefferia Oudemans, 1906

- H. gilvipes (C.L. Koch, 1839): 8, 13, 39, 40, $42,47,49,61$

LIACARIDAE Sellnick, 1928

Adoristes Hull, 1916

- A. ovatus (C.L. Koch, 1840): 2, 3, 8, 9, 21, 24, 29, 30a, 31, 31a, 32, 34a, 47, 50, 53, 66

- A. poppei (Oudemans, 1906): 1, 3, 4, 8, 9, 11, 29, 30a, 31, 31a, 32-34, 34a, 53, 55, 66, 7173 
Birsteinius Krivolutsky, 1965

- B. clavatus Krivolutsky, 1965: 4

- B. perlongus Krivolutsky, 1965: 22

Dorycranosus Wooley, 1969

- D. moraviacus (Willmann, 1954): 4, 11, 22, 24,58

- D. splendens (Coggi, 1898): 3, 4, 34, 34a

Liacarus Michael, 1898

- L. brevilamellatus Mihelčič, 1955: 2, 7, 8,23, 48

- L. coracinus (C.L. Koch, 1840): 3-5, 7-9, 19, 22-25, 27, 29, 30a, 31, 31a, 33, 34, 34a, $35,39-47,52,53,55,55 \mathrm{a}, 62-65,68,71,74$, 75

- L. cuspidatus Mihelčič, 1954: 7-9, 13, 23, 24, 27, 29, 30a, 32, 34a, 41, 44, 45, 47, 50, 53

- L. gracilis Mihelčič, 1954: 38, 47

- L. lencoranicus Krivolutsky, 1967: 23, 24, 26, 29, 31a, 32, 33, 34a, 39, 48, 53, 56

- L. nitens (Gervais, 1844): 19, 21, 22, 24, 40, 43, 53

- L. punctulatus (C.L. Koch, 1844): 3

- L. subterraneus (C.L. Koch, 1844): 59

- L. tremellae (Linné, 1761): 8, 26, 31a, 32, 38, 42, 45-47, 50, 53, 66

- L. vombi Dalenius, 1950: 21, 23, 27, 29, 31, 31a, 32, 33, 34a, 43, 46, 47, 49, 50, 53, 53a, 65

- L. xylariae (Schrank, 1803): 7-9, 47

XeNiLLIDAE Woolley \& Higgins, 1966

Xenillus Robineau-Desvoidy, 1839

- X. clypeator Robineau-Desvoidy, 1839: 4

- X. discrepans Grandjean, 1936: 74

- X. latus (Nicolet, 1885): 38, 40

- X. tegeocranus (Hermann, 1804): 3, 4, 8, 13, $15,16,23-26,29,30 \mathrm{a}, 33,34,34 \mathrm{a}, 35,38$, $39,42,43,45,47,48,51,55,55 \mathrm{a}, 56-58,66$, $72,74,75$

\section{ASTEGISTIDAE Balogh, 1961}

Astegistes Hull, 1916

- A. pilosus (C.L. Koch, 1840): 3, 4, 43

Cultroribula Berlese, 1908

- C. bicultrata Berlese, 1908: 8, 13, 38, 64, 72

- C. confinis Berlese, 1908: 21, 51

- C. falcata Evans, 1952: 71

- C. juncta (Michael, 1885): 3, 4

- C. lata Aoki, 1961: 3, 4, 9
- C. microdentata Krivolutsky, 1962: 3,4

- C. trifurcata var. rotundata Krivolutsky, 1962: 38

Furcoribula Balogh, 1943

- F. furcillata (Nordenskiöld, 1901): 3, 4, 40

METRIOPPIIDAE Balogh, 1943

Ceratoppia Berlese, 1908

- C. bipilis (Hermann, 1804): 2-5, 8, 9, 13, 15, 16, 21-24, 27-29, 31, 32, 34a, 38-43, 45-49, $53,53 \mathrm{a}, 56,65,66,71,72,74,75$

- C. quadridentata (Haller, 1882): 2, 8, 9, 13, $19,21,23,24,26,28-30,31 \mathrm{a}, 32,33,34 \mathrm{a}$, $38,39,41-48,65,66,74,75$

- C. sexpilosa Willmann, 1938:29, 53

Metrioppia Grandjean, 1931

- M. helvetica Grandjean, 1931: 2, 39, 53,53a

GustaviIDAE Oudemans, 1900

Gustavia Kramer, 1879

- G. fusifera (C.L. Koch, 1841): 39, 71, 74

- G. microcephala (Nicolet, 1855): 3-5, 7, 13, $21,23,24,26,31 \mathrm{a}, 42,45,46,48,57,66$

CARABOdidae C.L. Koch, 1837

Carabodes C.L. Koch, 1836

- C. areolatus Berlese, 1916: 2-4, 13, 30a, 31, 32, 34, 38, 39, 51, 58, 71, 74, 75

- C. coriaceus C.L. Koch, 1836: 4, 8, 13, 17, $32,34,42,46,53,57,71,75$

- C. femoralis (Nicolet, 1855): 23, 28, 34, 47, 57, 58, 61, 65, 71, 74

- C. femoralis var. rugosior Berlese, 1916: 8, $13,19,21,23,24,29,31$ a, 32, 43, 45, 46, 48, $51,55,66$

- C. forsslundi Sellnick, 1953: 8, 13,30a, 57

- C. labyrinthicus (Michael, 1879): 1, 8, 13, $21,23,24,27,29,32,33,34 a, 38,45-48,50$, $53,65,71,75$

- C. marginatus (Michael, 1884): 5, 7-9, 15, $16,19,23,25,27,29,31 \mathrm{a}, 32,34,34 \mathrm{a}, 46$ $49,53,57,65,71$

- C. minusculus Berlese, 1923: 1-4, 53, 64, 74

- C. ornatus Storkán, 1925: 71

- C. reticulatus Berlese, 1913: 3, 4, 34, 34a, 55, 55a, 64, 71, 74, 75

- C. rugosior Berlese, 1916: 71, 74, 75

- C. subarcticus Trägårdh, 1902: 4, 53, 58, 71, 74,75 
TECTOCEPHEIDAE Grandjean, 1954

Tectocepheus Berlese, 1913

- T. alatus Berlese, 1913: 32, 53

- T. knullei Vaněk, 1960: 13, 21

- T. minor (Berlese, 1903): 13, 23, 47, 48

- T. sarekensis (Trägårdh, 1910): 1-3, 8, 12, $13,32,38,42,48,53,66,68,71-74$

- T. velatus (Michael, 1880): 3, 4, 7-9, 11, 13, $18,19,21-31,31$ a, 32-34, 34a, 38, 42-49, 51, 53, 53a, 55, 55a, 56-62, 65, 66, 69, 71, 74,75

\section{OTOCEPHEIDAE Balogh, 1961}

Dolicheremaeus Jacot, 1938

- D. georgii Bulanova-Zakhvatkina, 1967: 38

- D. montanus Krivolutsky, 1971: 4

\section{OPPIDAE Grandjean, 1954}

Berminiella Balogh, 1983

- B. bicarinata Paoli, 1908: 2, 4, 8, 9, 13, 21, 23, 24, 28, 29, 30a, 31, 31a, 32, 45-51, 53, $54,57,65,66$

Cosmoppia Balogh, 1983

- C. ornata (Oudemans, 1900): 2-4, 7-9, 13, 19, 21, 23, 24, 26-29, 30a, 31, 31a, 32, 33, $34 \mathrm{a}, 38,42-50,53,54,64-66,71,74,75$

Ctenoppiella Gordeeva \& Karppinen, 1988

- C. fallax (Paoli, 1908): 3, 4, 7, 8, 46, 53, 61, 71

- C. globosa (Mihelčič, 1956): 8, 9, 13, 18, 21, $23,24,26,27,29,31,31 \mathrm{a}, 32,33,34 \mathrm{a}, 43$, 45-47, 49-51, 53, 54, 65, 66

- C. media (Mihelčič, 1965): 2, 13, 31a, 32, 34, $34 \mathrm{a}, 54,65$

- C. obsoleta (Paoli, 1908): 3, 4, 25, 28, 34, 38, 47, 53, 63, 64, 71, 74

C. subpectinata (Oudemans, 1901): 3, 47, 53, 57, 71, 72, 74, 75

- C. tuberculata (Bulanova-Zakhvatkina, 1964): 4, 27, 34, 34a, 53, 56, 74

Hypogeoppia Subias, 1982

- H. jannae (Sellnick, 1961): 3, 4, 34

- H. sigma var. conjuncta (Strenzke, 1951): 13, 31, 31a, 32, 47, 49

Microppia Balogh, 1983

- M. minus (Paoli, 1908): 3, 4, 8, 9, 13, 23, 26, $28,32,33,34 \mathrm{a}, 38,43,44,46,51,53,65$

- M. minutissima (Sellnick, 1950): 6, 48, 71
Multioppia Hammer, 1961

- M. glabra (Mihelčič, 1955): 6, 23, 24, 26, 27, 29, 31, 31a, 32, 41-43, 43a, 45, 46, 48, 50, $53,54,56-58,65,66,74,75$

- M. laniseta Moritz, 1966: 71

Oppia C.L. Koch, 1836

- O. chitinophincta Kulijev, 1962: 27, 29

- O. concolor (C.L. Koch, 1844): 4, 61

- O. furcata (Willmann, 1928): 38, 41, 47, 55, 74

- O. manifera Hammer, 1952: 34, 53

- O. minuta Bulanova-Zakhvatkina, 1964: 3

- O. nitens C.L. Koch, 1836: 3, 4, 13, 26, 31, 34, 34a, 38, 39, 41, 42, 46, 48, 67

- O. paradecipiens Kulijev, 1962: 4, 8, 9, 2124, 27-29, 30a, 31, 31a, 32, 33, 34a, 38, 42, 43, 45-48, 50, 53, 54, 55a, 56-59, 65, 66

- O. quadrimaculata Evans, 1952: 74

- O. splendens (C.L. Koch, 1840): 4, 55

- O. translamellata (Willmann, 1923): 30a, 38 Oppiella Jacot, 1937

- O. bicarinata (Paoli, 1908): 71, 75

- O. confinis (Paoli, 1908): 74

- O. falcata (Paoli, 1908): 34, 56, 57, 71

- O. loksai Schalk, 1966: 74

- O. marginedentata (Strenzke, 1951): 74

- O. maritima (Willmann, 1929): 8, 26, 28, 29, 31, 31a, 39, 42, 46, 47, 71

- O. neerlandica (Oudemans, 1900): 2, 3, 26, 27, 29, 31, 31a, 32, 47-49, 53, 54, 61, 65, 66, 71

- O. nova (Oudemans, 1902): 2-4, 7-9, 13, 18, $19,21,23,25,26,28,31,31 \mathrm{a}, 32-34,34 \mathrm{a}$, $38,39,42-48,51,53,55-62,65,66,68,71$, 74,75

- O. uliginosa (Willmann, 1919): 74

- O. unicarinata (Paoli, 1908): 3, 4, 13, 34, 53, $71,74,75$

Quadroppia Jacot, 1939

- Q. maritalis Lions, 1982: 74

- Q. paolii Woas, 1986: 74, 75

- Q. quadricarinata (Michael, 1885): 2, 3, 8, 9, 13, 21, 23, 24, 26, 28, 29, 31, 31a, 32, 33, 38, $39,43-47,49-51,53,55-57,65,66,71,74$, 75

Ramusella (Insculptoppia) Subias, 1980

- $R$. clavipectinata (Michael, 1885): 4, 9, 24, 34, 34a, 55-58

- R. insculpta (Paoli, 1908): 34a, 49

Ramusella (Rectoppia) Subias, 1980

- R. mihelcici (Perez-Inigo, 1964): 4, 57 
SUCTOBELbiDAE Grandjean, 1954

Allosuctobelba Mortiz, 1970

- A. grandis (Paoli, 1908): 8, 57, 75

Rhynchobelba Willmann, 1953

- R. inexpectata Willmann, 1953: 34

Suctobelba Paoli, 1908

- S. aliena Moritz, 1970: 29, 46, 56-58

- S. altvateri Mortiz, 1970: 74

- S. granulata van der Hammen, 1952: 71

- S. lapidaria Moritz, 1970: 21, 24, 29, 46

- S. regia Moritz, 1970: 51,71

- S. reticulata Moritz, 1970: 8, 27, 32, 53

- S. scalpelata Moritz, 1970: 29

- S. secta Mortiz, 1970: 13, 64

- S. sorrentensis Hammer, 1961: 4

- S. trigona (Michael, 1888): 8, 9, 13, 21-24, 26-29, 30a, 31, 31a, 32, 33, 34a, 38, 39, 42$51,53,54,65,66,68,71,74,75$

Suctobelbella Jacot, 1937

- S. acrana Moritz, 1970: 6, 29, 71, 74, 75

- S. acutidens (Forsslund, 1941): 8, 28, 38, 46, $47,71,75$

- S. alloenasuta Moritz, 1971: 4, 6, 13, 21, 23, 29, 31, 31a, 45-47, 49, 53, 54, 64, 66

- S. amurica (Krivolutsky, 1966): 8, 13, 29

- S. bella (Berlese, 1902): 4, 8, 64

- S. duplex (Strenzke, 1950): 8, 13, 23, 26, 27, 29, 31, 31a, 32, 33, 44-48, 50, 53, 66

- S. falcata (Forsslund, 1941): 6, 13, 32, 33, 47, 48, 51, 61, 71

- S. forsslundi (Strenzke, 1950): 8, 38, 46, 47, 75

- S. hammeri (Krivolutsky, 1965): 6, 28, 29, 31a, 32, 38, 47, 49, 51, 53, 64, 65

- S. latirostris (Strenzke, 1950): 1, 8, 27, 29, 31a, 45, 47

- S. longirostris (Forsslund, 1941): 8, 54, 64, 71

- S. macrodon Mihelčič, 1956: 8, 13

- S. nasalis (Forsslund, 1941): 8, 29, 31a, 71

- S. opistodentata (Golosova, 1970): 57

- S. palustris (Forsslund, 1953): 8, 29, 30a, 31, 31a, 47, 48, 53, 65

- S. paracutidens Mahunka, 1983: 74

- S. perforata (Strenzke, 1950): 6, 27, 28, 31a, 32, 38, 49, 53, 65, 71

- S. perpendiculata (Forsslund, 1958): 51, 53, 57,65

- S. promineus (Moritz, 1966): 29, 34, 47

- S. sarekensis (Forsslund, 1941): 8, 71
- S. similis (Strenzke, 1950): 2, 8, 9, 31, 31a, 34a, 51, 53, 71

- S. singularis (Strenzke, 1950): 34, 34a

- S. subcornigera (Forsslund, 1941): 8, 27, 28, 31a, 47, 57, 58, 71, 74

- S. subtrigona (Oudemans, 1916): 2-4, 8, 21, 26, 31a, 34, 34a, 45, 47, 53, 62, 71, 74

- S. tatarica (Krivolutsky, 1968): 31

- S. tuberculata Strenzke, 1950: 1, 8, 9, 13, 18, 26, 29, 31, 31a, 32, 39, 42-46, 48, 50, 51, 53, 65

- S. vera (Moritz, 1964): 8, 9, 13, 21, 23, 24, 27, 30a, 32, 33, 45-47, 51, 66

AutoGnetidae Grandjean, 1960

Autogneta Hull, 1916

- A. longilamellata (Michael, 1888): 3, 4, 34, 34a, 53, 57, 74, 75

- A. parva Forsslund, 1947: 53

- A. trägårdhi Forsslund, 1947: 2, 4, 8, 23, 29 , $43,46,48,53,65$

- A. willmanni (Dyrdowska, 1929): 4, 28, 32, 46, 47

Conchogneta Grandjean, 1963

- C. delacarlica (Forsslund, 1947): 8, 9, 13, 21, 23, 24, 26, 27, 29, 30, 31a, 39, 43-51, 53, $54,57,58,65,66$

\section{Caleremaeidae Grandjean, 1965}

Caleremaeus Berlese, 1910

- C. monilipes (Michael, 1882): 25, 34, 34a, $53,71,75$

THYRISOMIDAE Grandjean, 1953

Banksinoma Oudemans, 1900

- B. lanceolata (Michael, 1888): 3, 4, 66, 71, 74

Oribella Berlese, 1908

- O. alpestris (Willmann, 1929): 2, 49, 53, 53a, 56

- O. paoli Oudemans, 1913: 4, 8, 9, 22-24, 31, 31a, 32, 43, 46-49, 53, 53a, 60, 61, 65, 71, 74

- O. pectinata (Michael, 1885): 3, 4, 63

HyDROZETIDAE Grandjean, 1954

Hydrozetes Berlese, 1902

- H. confervae (Schrank, 1781): 4

- H. lemnae Coggi, 1899: 3, 4 
LIMNOZETIDAE Grandjean, 1954

Limnozetes Hull, 1916

- L. sphagni (Michael, 1884): 4

CymbaeremaeIDAE Sellnick, 1928

Cymbaeremaeus Berlese, 1896

- C. cymba (Nicolet, 1855): 3, 4, 71

Scapheremaeus Berlese, 1910

- S. palustris Sellnick, 1924: 52

MiCREREMIDAE Grandjean, 1954

Micreremus Berlese, 1908

- M. brevipes (Michael, 1888): 28, 48

- M. gracilior Willmann, 1931: 40, 69

LiCNEREMAEIDAE Grandjean, 1931

Licneremaeus Paoli, 1908

- L. licnophorus (Michael, 1888): 74

SCUTOVERTICIDAE Grandjean, 1954

Scutovertex Michael, 1879

- S. minutus (C.L. Koch, 1936): 3, 4, 25, 29, 30, 34, 34a, 48, 53

- S. punctatus Sitnikova, 1975: 4, 34

- S. rugosus Mihelčič, 1975: 4

- S. sculptus Michael, 1879: 72

- S. serratus Sitnikova, 1975: 4

ORIBATULIDAE Thor, 1929

Dometorina Grandjean, 1951

- D. plantivaga (Berlese, 1896): 32

Eporibatula Sellnick, 1928

- E. rauschenensis (Sellnick, 1908): 34

Hemileius Berlese, 1916

- H. initialis (Berlese, 1908): 65, 71, 72, 74, 75

Liebstadia Oudemans, 1906

- L. humerata Sellnick, 1928: 3, 34, 75

- L. similis (Michael, 1888): 3, 4, 6, 8, 9, 15, 16, 22-24, 27, 29, 31, 31a, 34a, 38, 46, 47, $49,53,65$

Lucoppia Berlese, 1908

- L. lucorum (C.L. Koch, 1840): 4

Oribatula Berlese, 1896

- O. pallida Banks, 1906: 3, 4, 27, 31, 31a, 32, 34, 34a, 40, 46, 53, 60, 65

- O. pannonica Willmann, 1949: 3, 55

- O. tibialis (Nicolet, 1855): 1-5, 8, 9, 15, 16,
$21,23,24,27-29,31,31 \mathrm{a}, 32-34,34 \mathrm{a}, 38$, $42,43,45-49,51,53,56,65,66,71-74$

Paraleius Travé, 1960

- P. leontonychus (Berlese, 1910): 71

Scheloribates Berlese, 1908

- S. confundatus Sellnick, 1928: 23, 27, 29, 31, 31a, 32, 43, 46, 47, 49, 51, 53, 53a, 65, 66

- S. labyrinthicus Jeleva, 1962: 53, 71, 72

- S. laevigatus (C.L. Koch, 1836): 3, 4, 7-9, 13, 17, 19, 21-26, 30, 31a, 33, 34, 34a, 35, $38,39,42-44,52,53,55,55 a, 62-64,66,67$, 71,72

- S. latipes (C.L. Koch, 1844): 2-5, 8, 9, 1517, 21, 23, 24, 26, 27, 29, 30a, 31, 31a, 32, $34 a, 39,40,42-44,46,47,49,53,53 a, 65$, $66,71,75$

- S. pallidulus (C.L. Koch, 1840): 3, 34, 55, 71,74

Zygoribatula Berlese, 1917

- Z. exilis (Nicolet, 1855): 9, 40, 48, 71, 72, 75

- Z. frisiae (Oudemans, 1900): 3, 4, 47, 55

- Z. microporosa Bulanova-Zakhvatkina, 1967: 4,61

- Z. terricola van der Hammen, 1952: 4

- Z. vera Bulanova-Zakhvatkina, 1967: 3, 4

HAPLOZETIDAE Grandjean, 1936

Haplozetes Willmann, 1935

- H. vindobonensis Willmann, 1935: 2, 23, 29, $42,53,65$

- H. vindobonensis var. curtipilus Kunst, 1977: 73

Peloribates Berlese, 1908

- P. europaeus Willmann, 1935: 3, 4, 43, 47

- P. longipilosus Csiszar, 1962: 4

Protoribates Berlese, 1908

- P. badensis Sellnick, 1928: 9, 13, 21, 23, 24, 26, 34a, 39, 43, 46, 51, 66

- P. capucinus (Berlese, 1908): 31a, 34, 38, 39, $51,67,72$

- P. longior Berlese, 1908: 47

- P. monodactylus (Haller, 1884): 4, 22, 24, 34, 34a, 56-58

- P. pannonicus Willmann, 1951: 75

- P. variabilis Rajski, 1958: 4, 7, 8, 28, 47, 55, 67

Xylobates Jacot, 1929

- X. lophotrichus (Berlese, 1904): 4, 26, 30a, 31a, 39, 41, 42, 47, 48, 50

- X. novus Willmann, 1953: 34a, 39 


\section{Chamobatidae Grandjean, 1954}

Chamobates Hull, 1916

- C. borealis (Trägårdh, 1902): 2, 9, 13, 19, 21-24, 27, 29, 30a, 31a, 32, 33, 43, 44, 46$49,53,53 \mathrm{a}, 65,66,71,72,74$

- C. caucasicus Shaldybina, 1969: 53

- C. cuspidatiformis (Trägårdh, 1904): 18, 20, 68

- C. cuspidatus (Michael, 1884): 1, 2, 8, 9, 26, $38,47,68,71,73$

- C. dapauperatus (Berlese, 1882-1889): 8

- C. lapidarius (Lucas, 1849): 28, 38

- C. pusillus (Berlese, 1895): 71, 73, 75

- C. schützi (Oudemans, 1901): 53

- C. spinosus Sellnick, 1928: 3, 4, 13, 34, 34a, 55,72

- C. subglobosus (Oudemans, 1900): 5, 8, 9, $12,13,15,16,26,33,34 \mathrm{a}, 42,45$

- C. tricuspidatus Willmann, 1953: 71

- C. voigtsi (Oudemans, 1902): 2, 8, 9, 13,2124, 27-29, 31, 31a, 32, 33, 34a, 38, 44-47, $49-51,53,65-67,71-75$

\section{EUZETIDAE Grandjean, 1954}

Euzetes Berlese, 1908

- E. globulus (Nicolet, 1855): 3-5, 8, 9, 13, 15, $16,18,19,21-24,26,29,34,41,43,46,47$, 49, 55, 55a, 57, 66, 67, 71

- E. seminulum (O.F. Müller, 1776): 40

ZETOMIMIDAE Shaldybina, 1966

Zetomimus Hull, 1916

- Z. furcatus (Pearce \& Warburton, 1906): 4, 71,72

\section{Ceratozetidae Jacot, 1925}

Ceratozetella Shaldybina, 1966

- C. minima (Sellnick, 1928): 2, 48

- C. sellnicki (Rajski, 1958): 3, 4, 8, 47, 51, 55a, 57, 71, 73

- C. thienemanni (Willmann, 1943): 31, 32, 47, $53,65,67,71$

Ceratozetes Berlese, 1908

- C. bulanovae Kulijev, 1962: 4

- C. cuspidodenticulatus Kulijev, 1962:4a

- C. furcatus (Pearce \& Warburton, 1905): 3

- C. gracilis (Michael, 1884): 3, 4, 7-9, 19, 34, $38,71,73,74$
- C. laticuspidatus Menke, 1964: 71

- C. longispinus Mahunka \& Topercer, 1983: 74

- C. macromediocris Shaldybina, 1970: 8, 26, 31a, 42, 44, 48, 68

- C. mediocris Berlese, 1908: 3, 4, 8, 22-24, 26, 27, 30a, 31a, 34, 34a, 39, 42, 44, 46, 48, 51, 53, 59-63, 72, 74

- C. minutissimus Willmann, 1951:4, 53, 61

- C. piritus Grandjean, 1951: 8, 13, 23, 26, 27, $39,41,42,45,49,53,68$

Ceratozetoides Shaldybina, 1966

- C. cisalpinus (Berlese, 1908): 3, 4, 8, 67

- C. maximus (Willmann, 1953): 34

Diapterobates Grandjean, 1936

- D. humeralis (Hermann, 1804): 2, 8, 9, 13, $21,46,53,66,71$

Edwardzetes Sellnick, 1928

- E. edwardsii (Nicolet, 1855): 5, 31, 31a, 32, $40,49,53,65,71$

Fuscozetes Sellnick, 1928

- F. fuscipes (C.L. Koch, 1844): 2, 5, 8, 9, 15, $16,53,71$

- F. setosus (C.L. Koch, 1840): 8, 21, 23, 27, 29, 30a, 31, 32, 39, 46, 47, 49, 50, 53, 53a, 65,71

Globozetes Sellnick, 1928

- G. tricuspidatus (Willmann, 1953): 23

Latilamellobates Shaldybina, 1971

- L. incisellus (Kramer, 1897): 3, 4, 29, 31a, 53,65

Melanozetes Hull, 1916

- M. longisensillus Schweizer, 1956: 31a

- M. meridianus Sellnick, 1928: 31a

- M. mollicomus (C.L. Koch, 1840): 9, 11, 15, $16,21,23,31,31 \mathrm{a}, 32-34,34 \mathrm{a}, 40,43,47$, $50,51,53,53 \mathrm{a}, 56-58,66,71,74,75$

- M. mollisimilis Schweizer, 1956: 4, 23, 30a, 31, 31a, 32, 45, 56, 66

Oromurcia Thor, 1930

- O. sudetica Willmann, 1939: 32, 49, 53, 65

Sphaerozetes Berlese, 1885

- S. orbicularis (C.L. Koch, 1836): 17, 31a, 53, 65

- S. piriformis (Nicolet, 1855): 5, 9, 15, 16

- S. tricuspidatus Willmann, 1923: 22, 24

Trichoribates Berlese, 1910

- T. longipilis Willmann, 1951:31, 31a, 46, 49, 53, 65

- T. monticola (Trägårdh, 1910): 34 
- T. novus (Sellnick, 1928): 8

- T. numerosus (Sellnick, 1924): 5, 9, 15, 16

- T. trimaculatus (C.L. Koch, 1836): 3-5, 16, $34 \mathrm{a}, 40$

\section{MyCOBATIDAE Grandjean, 1954}

Minunthozetes Hull, 1916

- M. pseudofusiger (Schweizer, 1922): 2, 8, 9, 18, 21, 23, 24, 26-29, 30a, 31, 31a, 32, 34a, $38,39,42,43,45-51,53,53 \mathrm{a}, 65-67,69,74$

- M. semirufus (C.L. Koch, 1841): 2, 8, 9, 11, 13, 18, 21-24, 26-29, 30a, 31, 31a, 33, 38, 39, 42, $43,45,46,48,50,53,53 \mathrm{a}, 66,71,73,74$

- M. tarmani Feider, Vasiliu \& Calugar, 1971: 57 Mycobates Hull, 1916

- M. bicornis Strenzke, 1954: 1,65

- M. carli (Schweizer, 1922): 27, 29, 32, 49, 53,65

- M. parmeliae (Michael, 1884): 53

- M. patrius Shaldybina, 1970: 32

- M. tridactylus Willmann, 1929: 3, 4, 70

Punctoribates Berlese, 1908

- P. hexagonus Berlese, 1908: 22, 49

- P. punctum (C.L. Koch, 1839): 1-4, 8, 9, 13, 19, 21, 23, 24, 27, 29, 31, 31a, 34, 41, 43-48, $55,59-63,66,67,72,74,75$

- P. sellnicki (Willmann, 1928): 7, 8,34a

- P. zachvatkini Shaldybina, 1969: 24, 26,30a, 31a, 39, 44, 45, 48

MochlozETIDAE Grandjean, 1960

Podoribates Berlese, 1908

- P. gratus (Sellnick, 1921): 3

PELOPIDAE Ewing, 1917

Eupelops Ewing, 1917

- E. acromios (Hermann, 1804): 2-4, 12, 13, 19, 23, 26, 29, 31, 31a, 32, 34, 34a, 42, 45, 53, 54, 55a, 56, 71

- E. auritus (C.L. Koch, 1840): 28, 38

- E. bilobus Sellnick, 1928: 1,34

- E. caucasicus Sitnikova, 1975: 9, 23

- E. nepotulus Berlese, 1917: 24, 72, 74

- E. occultus (C.L. Koch, 1936): 2, 9, 10, 21, 23, 24, 26, 31a, 39, 40, 43, 44, 47, 49, 66, 71

- E. planicornis (Schrank, 1803): 38

- E. plicatus (C.L. Koch, 1836): 1, 3, 4, 8, 12, 13, 19, 21-24, 27, 29, 31, 31a, 32, 34a, 39, 42, 45, $46,48,49,53,53 \mathrm{a}, 54,65,66,71,74,75$
- E. subuliger Berlese, 1917: 29, 30a, 31, 31a, 32, 34, 34a, 40, 49, 53, 65, 66, 71

- E. tardus (C.L. Koch, 1836): 3, 7

- E. torulosus (C.L. Koch, 1840): 1, 2, 8-10, 12, 13, 19, 21, 23, 28, 30a, 31, 31a, 32, 33, 34a, 37a, 47, 53, 54, 65, 66, 71, 72, 74

Peloptulus Berlese, 1908

- P. phaenotus (C.L. Koch, 1844): 2-4, 7, 21, 22, 34, 34a, 53, 65

\section{ORIBATELLIDAE Jacot, 1925}

Ophidiotrichus Grandjean, 1953

- O. connexus (Berlese, 1904): 13, 23, 27, 34a, $38,45,46,51,66$

- O. connexus var. borussicus (Sellnick, 1908): 47, 74, 75

Oribatella Banks, 1895

- O. angulosa Csiszar, 1962: 2, 8, 21, 23, 24, 32, 34a, 43, 48, 49, 53, 66-68

- O. berlesei Michael, 1898: 13, 21-24, 28, 31, $40,46,47,49,53,57,66,72,74$

- O. calcarata (C.L. Koch, 1836): 4, 5, 9, 15, 16, 40, 57, 71, 73-75

- O. dudichi Willmann, 1938: 2, 19, 23, 28, 31a, 34a, 43, 46, 47, 49, 53, 65, 74

- O. enthricha Berlese, 1908: 15, 16

- O. kurchevi Krivolutsky, 1974: 31a, 40, 46, $47,49,50,53$

- O. meridionalis Berlese, 1908: 3, 4, 55, 58

- O. ornata (Coggi, 1900): 7, 8, 19, 31a, 32, $46,47,53,60$

- O. quadricornuta (Michael, 1884): 5, 58, 71

- O. reticulata Berlese, 1916: 55a

- O. sexdentata Berlese, 1916: 4

Tectoribates Berlese, 1910

- T. ornatus (Schuster, 1958): 3, 4, 6

TegoribatidAe Grandjean, 1954

Lepidozetes Berlese, 1910

- L. singularis Berlese, 1910: 22, 29, 31, 31a, $32,46-48,53$

Scutozetes Hammer, 1952

- S. lanceolatus Hammer, 1952: 19, 22

ACHIPTERIIDAE Thor, 1929

Achipteria Berlese, 1885

- A. coleoptrata (Linné, 1746): 1, 2, 4, 5, 7-16, 18, 19, 21-27, 29, 30a, 31a, 32, 33, 34a, 37a, $38-50,53,54,56-59,65-68,71-74$ 
- A. italica (Oudemans, 1913): 21-23, 27, 29, 32, 46, 53, 53a, 65, 66

- A. nitens (Nicolet, 1855): 1, 2, 4, 8-10, 12, 13, $18-20,22-24,30 a, 33,34,34 a, 37 a, 38,39,41-$ $43,45,50,53,54,56-59,65,66,72,74,75$

- A. oudemansi Hammer, 1952: 57, 65

- A. sellnicki van der Hammen, 1952: 4

- Anachipteria Grandjean, 1935

- A. deficiens Grandjean, 1935: 4, 73

- A. latitecta (Berlese, 1908): 4, 13, 34, 40, 43, 44, 46, 47, 56, 65

Parachipteria van der Hammen, 1952

- P. bella (Sellnick, 1928): 34, 34a, 40, 55, 59

- P. nivalis (van der Hammen, 1952): 4, 31a, 53, 59

- P. patovicus (Oudemans, 1913): 53

- P. punctata (Nicolet, 1855): 1-4, 7-14, 1719, 21, 23-25, 27-29, 31a, 32, 34, 34a, 38, 40, 42, 43, 46-49, 53-55, 57, 65-68, 74

- P. willmanni (van der Hammen, 1952): 4, 12, 25, 53-57, 65, 71, 74, 75

\section{PARACALUMMIDAE Grandjean, 1936}

Neoribates Berlese, 1914

- N. neglectus Willmann, 1953: 71

Protokalumma Jacot, 1929

- P. aurantiaca (Oudemans, 1913): 4, 8, 9, 13, 21-24, 26, 31a, 43, 61, 66

\section{GALUMNIDAE Jacot, 1925}

Acrogalumna Grandjean, 1956

- A. longipluma (Berlese, 1904): 5, 8, 9, 13, $15,21,27,29,46,49,68,71,74,75$

Allogalumna Grandjean, 1936

- A. crassivlava (Berlese, 1904): 8

Galumna von Heyden, 1826

- G. alata (Hermann, 1804): 26

- G. dimorpha Krivolutskaja, 1952: 4

- G. elimata (C.L. Koch, 1841): 5, 8, 71

- G. flagellata Willmann, 1925: 4

- G. lanceata Oudemans, 1900: 1-4, 7-9, 12, $17,18,23,26,29,34,39,42,43,47,48,53$, $55,57,71$

- G. obvius (Berlese, 1914): 3-5, 8-10, 12, 13, 15, 21-25, 31a, 42, 43, 54, 66, 67

- G. rossica Sellnick, 1926: 3, 4

- G. tarsipennata Oudemans, 1913: 1, 71, 74

Pergalumna Grandjean, 1936

- P. altera (Oudemans, 1915): 2, 12, 21-23, 29 , 30a, 31, 31a, 37a, 40, 53, 53a, 54, 65, 71
- P.formicaria (Berlese, 1914): 10, 53

- P. myrmophila (Berlese, 1915): 10, 53, 69

- P. nervosa (Berlese, 1914): 1-6, 9, 15, 16, $18,23,60,71,74$

Pilogalumna Grandjean, 1956

- P. allifera (Oudemans, 1919): 3, 4, 34, 34a

- P. allifera var. montana Grishina \& Sergienko, 1978: 37a

- P. ornatula Grandjean, 1956: 4

Acknowledgement. We are grateful to Mr. Pekka Vilkamaa for checking the translation and transliteration of the Russian references.

\section{References}

Balogh, J. 1972: The Oribatid genera of the world. Budapest. 188 pp.

Bulanova-Zakhvatkina, E. М. (Буланова-Захваткина, Е. M.) 1965: [Diagnostics of the species in the genus Metabelba Grandjean, 1936 (Oribatei, Damaeidae)]. (In Russian) - Zool. Zh. 44:1333-1344.

- 1967: [Oribatid mites]. (In Russian) - Vyshaya Shkola. 253 pp.

- 1975: [Superfamilies Belboidea, Eremaeoidea, Carabodoidea, Oribatuloidea]. (In Russian) - In: Gilarov, M. S. \& Krivolutsky, D. A. (Гиларов, М. C. \& Криволутский, Д. А.) (eds.), [Identification of soil mites, Sarcoptiformes]: 120-143, 158-161, 184-190, 257-275. Izd. Nauka, Moscow.

Dyrdowska, M. 1931: Studja nad Fauna nechowcow (Oribatidae) Polski. - Spraw. Kom. Fiz., Krakowie t. 65:121-144.

Golosova, L., Karppinen, E. \& Krivolutsky, D. A. 1983: List of oribatid mites (Acarina, Oribatei) of northern palaearctic region. II. Siberia and the Far East. - Acta Entomol. Fennica 43:1-14.

Grishina, L. G. \& Sergienko, G. D. (Гришина, Л. Г. \& Сергиенко, Г. Д.) 1978: [Winged oribatid mites (Oribatei, Galumnoidea) of Ukraine]. (In Russian) Vestnik Zool. 3:48-52.

Karppinen, E. \& Krivolutsky, D. A. 1982: List of oribatid mites (Acarina, Oribatei) of northern palaearctic region. I. Europe. - Acta Entomol. Fennica 41:1-18.

Karppinen, E., Krivolutsky, D. A., Koponen, M., Kozlovskaja, L. S., Laskova, L. M. \& Viitasaari, M. 1979: List of subfossil oribatid mites (Acarina, Oribatei) of northern Europe and Greenland. - Ann. Entomol. Fennici 45:103-108.

Karppinen, E., Krivolutsky, D. A. \& Poltavskaja, M. P. 1986: List of oribatid mites (Acarina, Oribatei) of northern palaearctic region. III. Arid lands. - Ann. Entomol. Fennici 52:81-94.

Karppinen, E., Krivolutsky, D. A., Tarba, Z. M., Shtanchaeva, U. Ya. \& Gordeeva, E. W. 1987: List of ori- 
batid mites (Acarina, Oribatei) of northern palaearctic region. IV. Caucasus and Crimea. - Ann. Entomol. Fennici 53:119-137.

Kazakov, V. І. (Казаков, В. И.) 1981: [Studies on oribatid mites of the superfamily Oppioidea (Oribatei) in soils of some evergreen forests in western parts of USSR]. (Abstract, in Russian) - Problemy Pochvennoi Zoologii VII, Kiev: 88.

- 1985: [Oribatid mites of the family Oppiidae (Grandjean, 1954)]. (In Russian) - Katal. Mus. Fond. Zbirn. Nauk. Prats, Kiev: 49-53.

Krivolutsky, D. А. (Криволутский, Д. А.) 1975: [Superfamily Oribatelloidea, subgroup Ptyctima]. (In Russian) - In: Gilarov, M. S. \& Krivolutsky, D. A. (Гиларов, М. С. \& Криволутский, Д. А.) (eds.), [Identification of soil mites, Sarcoptiformes]: 326346, 366-380. Izd. Nauka, Moscow.

Krivolutsky, D. A., Karppinen, E. \& Golosova, L. 1984: The relict endemic fauna of oribatid mites (Acarina, Oribatei) of southern Siberia. - Ann. Entomol. Fennici 50:118-120.

Kulczynski, W. 1902: Species Oribatinarum (Oudems.) (Damaeinarum Michael) in Galicia collectae. - Rozp. Spraw. Posiend. Wydz., Mat. Przyr. A.U., 42B:1-50.

Kurcheva, G. F. (Курчева, Г. Ф.) 1970: [Oribatid mites of Zakarpatiya]. (In Russian) — In: [Oribatid mites (Oribatei) and their role in soil-forming processes]: 73-79. Vilnius.

- 1973: [Fauna of oribatid mites in different soil types in oak forests of the European part of the USSR]. (In Russian) - In: Ghilarov, M. S. (ed.), Ecology of soil invertebrates: 208-223. Nauka, Moscow.

Melamud, V. V. (Меламуд, В. В.) 1987: [Studies on oribatid mites (Acariformes, Oribatei) of Chivchinsk high mountains on the border of the Romanian Carpathians]. (Abstract, in Russian) - Problemy Pochvennoi Zoologii IX, Tbilisi: 182-183.

Polonchik, E. M. \& Fasulati, G. 1964 (Полончик, E. M. \& Фасулати, Г.) 1964: [Distribution of Oribatei in forest soils of Zakarpat region]. (In Russian) - In: Ecology of insects and other invertebrates in the Soviet Carpathians, Uzgorod: 74-75. Uzgorod Univ.

Sergienko, G. D. (Сергиенко, Г. Д.) 1984: A contribution to the studiy of lower oribatids (Oribatei, Macropylina) of the Ukrainian SSR fauna. (In Russian) - Vestnik Zool. 1884(5):31-35.

- 1985: A contribution to the study of oribatid genus Steganacarus (Oribatei, Phthiracaridae) of the Ukrain- ian fauna]. (In Russian with English summary) Vestnik Zool. 1985(3):30-37.

- 1987a: Macropylina (Oribatei) of the fauna of the Ukraine. (In Russian) — Vestnik Zool. 1987(2):3338.

- 1987b: Oribatid mites of the genera Phthiracarus and Archiphthiracarus (Oribatei, Phthiracaridae) of the Ukraine. Communication I. (In Russian with English summary) - Vestnik Zool. 1987(6):35-43.

Sevastanov, V. D. (Севастьанов, В. Д.) 1970: [Acarofauna of the forest ant Formica rufa L]. (In Russian) - In: Oribatids and their role in soil-forming processes: 143147. Vilnius.

Shaldybina, E. S. (Шалдыбина, Е. C.) 1981: [Sphaerozetid fauna of the Soviet Union (Oribatei, Sphaerozetidae)]. (In Russian) - In: Fauna, systematics, biology and ecology of helmints and their intermediate hosts: 99110. Gorsky Pedagogical Inst., Gorkii.

Sitnikova, L. G. (Ситникова, Л. Г.) 1975: [Superfamilies Nothroidea, Nanhermannoidea, Hermannielloidea, Cymbaeremaeoidea, Pelopoidea]. (In Russian) - In: Gilarov, M. S. \& Krivolutsky, D. А. (Гиларов, М. C. \& Криволутский, Д. А.) (eds.), [Identification of soil mites, Sarcoptiformes]: 71-111, 234-242, 320-326. Nauka.

Udvardy, M. D. F. 1975: World biogeographical provinces. - Int. Union Conserv. Nature National Res.. Occ. Paper 18. Map.

Yaroshenko, N. N. (Ярошенко, Н. Н.) 1978: [Oribatid fauna of the Ukrainian Polesya]. (In Russian) Vestnik Zool. 4:60-63.

Yavornitskii, V. I., Shaposhnikova, E. V. \& Melamud, V. V. (Яворнитский, В. И., Шапошникова, Е. В. \& Меламуд, В. В.) 1984: [Complexes of soil microarthropods in hornbeam-oak forests in upper bassein of the river Dnestr]. (In Russian) - Problemy Pochvennoi Zoologii VIII, Ashabad: 170-171.

Zgerskaya, E. V. (Згерская, Е. В.) 1980: [Studies on oribatid mites - vectors of cestodes in lowland meadows of Lvov region]. (In Russian) - 9. Konf. Ukrainsk. Parasitol. Obshch. Tes. Dokl. Kiev: 62-63.

Zgerskaya, E. V. \& Senyk, А. F. (Згерская, Е. В. \& Сенык, А. Ф.) 1975: [Studies on oribatid mites of the forest biocenoses in plain area of the Lvov region]. (In Russian) - Probl. Parasitol. Material VIII. Nauch. Konf. Parasitol. UkrSSR, Kiev. 186 pp.

Received 8.V.1990 